\title{
Striatal vessels receive phosphorylated tyrosine hydroxylase-rich innervation from midbrain dopaminergic
} neurons

\section{Domingo Afonso-Oramas ${ }^{1,2,3}$, Ignacio Cruz-Muros ${ }^{1,2,3}$, Javier Castro-Hernández 1,2, Josmar Salas-Hernández ${ }^{1,3}$, Pedro Barroso-Chinea ${ }^{1,2}$, Sonia García-Hernández ${ }^{4}$, José L. Lanciego ${ }^{3,5}$ and Tomás González-Hernández ${ }^{1,2,3}$ *}

${ }^{1}$ Department of Anatomy, Faculty of Medicine, University of La Laguna, La Laguna, Tenerife, Spain

${ }^{2}$ Biomedical Technologies Institute (ITB, CIBICAN), La Laguna, Tenerife, Spain

${ }^{3}$ Spanish Network of Neurodegenerative Diseases (CIBERNED), Madrid, Spain

${ }^{4}$ Department of Pathology, University Hospital of Canary Islands, Tenerife, Spain

${ }^{5}$ Center for Applied Medical Research (CIMA), University of Navarra, Pamplona, Spain

\section{Edited by:}

Javier Blesa, Columbia University, USA

\section{Reviewed by:}

Yoland Smith, Emory University, USA

Jose L. Labandeira-Garcia, University of Santiago de Compostela, Spain

${ }^{*}$ Correspondence:

Tomás González-Hernández, Department of Anatomy, Faculty of Medicine, University of La Laguna, 38207 La Laguna, Tenerife, Spain e-mail: tgonhern@ull.es
Nowadays it is assumed that besides its roles in neuronal processing, dopamine (DA) is also involved in the regulation of cerebral blood flow. However, studies on the hemodynamic actions of DA have been mainly focused on the cerebral cortex, but the possibility that vessels in deeper brain structures receive dopaminergic axons and the origin of these axons have not been investigated. Bearing in mind the evidence of changes in the blood flow of basal ganglia in Parkinson's disease (PD), and the pivotal role of the dopaminergic mesostriatal pathway in the pathophysiology of this disease, here we studied whether striatal vessels receive inputs from midbrain dopaminergic neurons. The injection of an anterograde neuronal tracer in combination with immunohistochemistry for dopaminergic, vascular and astroglial markers, and dopaminergic lesions, revealed that midbrain dopaminergic axons are in close apposition to striatal vessels and perivascular astrocytes. These axons form dense perivascular plexuses restricted to striatal regions in rats and monkeys. Interestingly, they are intensely immunoreactive for tyrosine hydroxylase (TH) phosphorylated at Ser19 and Ser40 residues. The presence of phosphorylated $\mathrm{TH}$ in vessel terminals indicates they are probably the main source of basal TH activity in the striatum, and that after activation of midbrain dopaminergic neurons, DA release onto vessels precedes that onto neurons. Furthermore, the relative weight of this "vascular component" within the mesostriatal pathway suggests that it plays a relevant role in the pathophysiology of PD.

Keywords: dopamine, midbrain, striatum, Ser 19, Ser 40, cerebral blood flow, Parkinson's disease

\section{INTRODUCTION}

DA is a neurotransmitter and neuromodulator involved in a wide range of brain functions including control of voluntary movements, reward-seeking behavior, cognitive processes and circuit formation during development (Schultz, 2007; Money and Stanwood, 2013; Morita et al., 2013). DA also exerts important actions in peripheral organs, with those being on the vascular dynamics control of particular relevance (Tayebati et al., 2011). Studies carried out in the 1970s and 1980s showed that DA and DA agonists have vasomotor effects on major extracerebral arteries and pial arterioles of the cortical surface, promoting a general increase in cerebral blood flow (Toda, 1976; Ingvar et al., 1983; Edvinsson et al., 1985). More recently, it has been shown that DA analogs also modify blood flow into discrete brain regions (Breiter et al., 1997; Marota et al., 2000), and that this effect is prevented by dopaminergic lesion (Chen et al., 1997; Nguyen et al., 2000; Jenkins et al., 2004). In sum, the involvement of DA in the regulation of regional cerebral blood flow is well-established nowadays. However, our knowledge on this issue is still fragmentary and relevant aspects have not been elucidated. For example, while some data suggest that vascular effects of DA require activation of postsynaptic $D_{1}$ DA receptors (Knutson and Gibbs, 2007), dopaminergic contacts have also been described in small vessels of the monkey frontal cortex (Krimer et al., 1998), and DA receptor expression has been found in cultured endothelial cells and astrocytes (Bacic et al., 1991; Bal et al., 1994; Zanassi et al., 1999; Choi et al., 2006), suggesting a direct DA effect on vessels and surrounding astrocytes. On the other hand, most interest in cerebral blood flow regulation has been focussed on the cerebral cortex, whereas mechanisms operating in deep brain centers, including the striatum, have been less investigated. It is noteworthy that in spite of the striatum being the main target of midbrain dopaminergic inputs, there is no evidence that striatal vessels and surrounding astrocytes 
receive midbrain dopaminergic contacts. The clarification of this question could contribute to a better understanding of metabolic and hemodynamic events associated with striatal processing and their role in the pathophysiology of Parkinson's disease (PD). This possibility has been explored here by using nigral injections of an anterograde tracer in combination with immunofluorescence for dopaminergic, vascular and astroglial markers, confocal microspcopy and dopaminergic lesion.

\section{MATERIAL AND METHODS}

Experiments were carried out on 16 male Sprague-Dawley rats (250-300 g; Charles River, L'Arbresle, France) and three male rhesus monkeys (Macaca fascicularis, 6-7 years old, $3.5-4.8 \mathrm{Kg}$ ). Nine rats were used for the injection of the anterograde neuronal tracer biotinylated dextran amine (BDA) in the dopaminergic cell groups of ventral midbrain, and seven rats for 6-hydroxydopamine (6-OHDA) lesion. The three monkeys were processed for immunofluorescence. Experimental protocols were approved by the Ethical committee of the University of La Laguna (Reference \# 091/010), and are in accordance with the European Communities Council Directive of 22 September $2010(2010 / 63 / \mathrm{EU})$ regarding the care and use of animals for experimental procedures.

\section{SURGICAL PROCEDURES}

BDA injections were performed according to Lanciego and Wouterlood (2006). A 10\% solution of BDA (biotin, dextran $10 \mathrm{kDa}$; Molecular Probes, Leiden, The Netherlands) in $10 \mathrm{mM}$ phosphate buffer $\mathrm{pH} 7.25$ was iontophoretically delivered in the midbrain dopaminergic formation $(1.2-1.8 \mathrm{~mm}$ lateral to midline, $5.7-6.0 \mathrm{~mm}$ posterior to bregma and $8.0-8.5 \mathrm{~mm}$ below the dura, according to Paxinos and Watson, 1998) using a glass micropipette (inner tip diameter 20-30 $\mu \mathrm{m}$ ) and a positivepulsed direct current ( $7 \mathrm{~s}$ on/off) for $7 \mathrm{~min}$. The micropipette was left in place for $5 \mathrm{~min}$ before removal. Animals were killed 1 week after injection.

A rat model based on the intracerebroventricular injection of 6-OHDA was used for dopaminergic lesion. This model causes bilateral degeneration of mesostriatal dopaminergic neurons, and a motor syndrome composed of hypokinesia, purposeless chewing and catalepsy (Rodríguez et al., 2001; Rodríguez-Díaz et al., 2001; González-Hernández et al., 2004). Rats were injected in the third ventricle (midline, $2 \mathrm{~mm}$ posterior to bregma and $8 \mathrm{~mm}$ below the dura, according to Paxinos and Watson, 1998) with vehicle $(0.9 \%$ saline solution with $0.3 \mu \mathrm{g} / \mu \mathrm{l}$ ascorbic acid, sham group, $n=3)$ or a single dose $(400 \mu \mathrm{g})$ of 6-OHDA (6hydroxydopamine hydrochloride, Sigma, St. Luis, MO; in $8 \mu \mathrm{l}$ of vehicle per injection; $1 \mu \mathrm{l} / \mathrm{min}, 6$-OHDA groups, $n=4$ ). Anesthesia, pre-surgery treatment and intraventricular injection protocols followed Rodríguez et al. (2001). Bearing in mind that the bilateral degeneration of DA-cells can cause adipsia and aphagia (Zigmond and Stricker, 1973), the intake of food and water was monitored following the 6-OHDA injection. No body weight loss was observed and rats were killed 2 weeks after injection.

\section{TISSUE PROCESSING}

Animals were deeply anesthetized with an overdose of sodium pentobarbital and transcardially perfused with heparinized ice-cold $0.9 \%$ saline ( $150 \mathrm{ml}$ in rats, 11 in monkeys) followed by $4 \%$ paraformaldehyde in phosphate buffer saline $0.1 \mathrm{M} \mathrm{pH} 7.4$ PBS; $300 \mathrm{ml}$ in rats and 2.51 in monkeys). The brains were then removed, the midbrain and forebrain blocks were stored in the same fixative at $4^{\circ} \mathrm{C}$ ( $8 \mathrm{~h}$ in rats and $18 \mathrm{~h}$ in monkeys), cryoprotected in a graded series of sucrose-PBS solutions and stored at $-80^{\circ} \mathrm{C}$ until processing. Coronal sections $(25 \mu \mathrm{m}$ in rats, $40 \mu \mathrm{m}$ in monkeys) were obtained with a freezing microtome, collected in parallel series and processed for single and double immunohistochemical labeling.

For detecting BDA-stained fibers, floating sections were immersed for $30 \mathrm{~min}$ in $3 \% \mathrm{H}_{2} \mathrm{O}_{2}$ to inactivate endogenous peroxidase, washed several times in PBS, and then incubated for $90 \mathrm{~min}$ in either ExtrAvidin-peroxidase (1:5000, Sigma) or Cy2conjugated ExtrAvidin (1:1000; Amersham, Buckinghamshire, England) and $0.3 \%$ TX-100 in PBS. In sections incubated in ExtrAvidin-peroxidase, stained fibers were visible after immersion for 5-10 $\mathrm{min}$ in $0.005 \% 3^{\prime}-3^{\prime}$-diamiobenzidine tetrahydrochloride (DAB, Sigma) and $0.001 \% \mathrm{H}_{2} \mathrm{O}_{2}$ in cacodylate buffer $0.5 \mathrm{~N}$, $\mathrm{pH} 7.6$.

Sections incubated in Cy2-conjugated ExtrAvidin were washed several times in PBS, and incubated for $60 \mathrm{~min}$ at room temperature (RT) in 4\% normal goat serum (NGS, Jackson ImmunoResearch, West Grove, PA) in PBS, and overnight in PBS containing 2\% NGS and one of the primary antibodies: mouse anti-tyrosine hyroxylase (TH) monoclonal antibody (Sigma, 1:12,000), rabbit anti-TH phosphorylated at Ser19 (THp19) polyclonal antibody (PhosphoSolutions, Aurora, CO, 1:2000), rabbit anti-THp31 polyclonal antibody (PhosphoSolutions, 1:600), rabbit anti-THp40 polyclonal antibody (PhosphoSolutions, 1:600), goat anti-dopamine transporter (DAT) polyclonal antibody (1:1000, Santa Cruz Biotechnology, Santa Cruz, CA), mouse anti-endothelial nitric oxide synthase (eNOS) monoclonal antibody (1:1000, Sigma), mouse anti-glial fibrilary acidic protein (GFAP) monoclonal antibody (1:2000, Sigma), or mouse anti-vimentin monoclonal antibody (1:400, Abcam, Cambrige, UK). Immunofluorescent labeling was visible after incubation for $3 \mathrm{~h}$ in Alexa Fluor 488-conjugated donkey anti-rabbit IgG (1:150; Molecular Probes, OR) and Rhodamine (TRITC) conjugated goat anti-guinea-pig IgG (1:100; Jackson ImmunoResearch), Lissamine Rhodamine-conjugated donkey anti-goat IgG (1:100; Jackson ImmunoResearch) or Lissamine Rhodamineconjugated goat anti-mouse IgG (1:100; Jackson ImmunoResearch) in PBS containing 1:200 NGS.

After several rinses, the sections were mounted on gelatinized slides, air dried, coverslipped with Vectashield (Vector), and examined under a confocal laser scanning microscopy system (Olympus FV1000, Hamburg, Germany) using appropriate filters. Sections were first examined using low-magnification lenses, and the areas of interest were analyzed at high magnification $(\times 63$ oil-immersion Plan-Apochromat objective lens, NA 1.4) at a resolution of $1024 \times 1024$ pixels, and acquired in Z-stack mode (10 $\mu \mathrm{m}$ total thickness, $6 \mathrm{z}$-steps). Selected high-resolution confocal images were then deconvoluted by using the MetaMorph 6.1r 0 software (Molecular Devices, Downingtown, PA). Deconvolution is a post-adquisition computational process for correcting the optical blur inherent to any image adquisition system, restoring 
the appearance of original image with the highest degree of confidence (Sibarita, 2005; Salin et al., 2008).

In addition, a quantitative analysis of double labeled terminals (THp19-DAT and THp40-DAT) was performed in high magnification images. Four striatal sections $100 \mu \mathrm{m}$ apart were randomly selected in five rats. Four vessel-centered $200 \mu \mathrm{m} \times 200 \mu \mathrm{m}$ square regions were also randomly selected in each section. Simple and double labeled terminals were counted within $100 \mu \mathrm{m}$ from the putative border of the vessel. Data are expressed as the percentage of double labeled terminals per section \pm standard error of the mean.

\section{RESULTS}

Striatal terminals were studied in rats whose BDA injection was restricted to the midbrain dopaminergic cell groups (substantia nigra pars compacta (SNC) and ventral tegmental area (VTA), Figure 1A). Cases with injection involving neighboring centers were excluded. Consistent with previous reports (Gerfen et al., 1987; Joel and Weiner, 2000), the regional distribution of striatal BDA positive fibers varied with the location of the injection site. Injections in VTA and/or the rostromedial region of the SNC provided a terminal field preferentially localized in the ventral striatum and the ventral part of the dorsal striatum, while after injections in the caudolateral region of the SNC, terminals spread out across the dorsal striatum.

Axons through the ventral and dorsal striatum showed collateral fibers emitting terminals in close apposition to the wall of blood vessels of diverse diameters (range 50-500 $\mu \mathrm{m}$ ). They usually arise in the form of individual branches that emit isolated perivascular endings (Figures 1C,E,G), but sparse perivascular terminal plexuses were also found (Figures 1B,D,F). Double labeling for BDA and vimentin (a marker of intermediate filaments present in vessels and astrocytes) or GFAP (an astroglial marker) confirmed the presence of BDA-positive terminal and "en passant" buttons abutted on the wall of striatal vessels (Figure 1H) and astroglial cells wrapping them (Figure 1I).

In order to confirm the dopaminergic nature of this projection, striatal sections of BDA-injected rats were further processed for $\mathrm{TH}$ (the rate-limiting enzyme in DA synthesis) and DAT. For $\mathrm{TH}$ immunohistochemistry, antibodies against the native (non-phosphorylated) as well as the phosphorylated forms of $\mathrm{TH}$ at serine 19 ( THp19), serine 31 (THp31) and serine 40 (THp40) were used. Immunochemistry for native $\mathrm{TH}$ resulted in a diffuse striatal staining (Figure 2A), making it difficult to identify double TH-BDA positive axons. Consistent with previous reports supporting the idea that under basal conditions only a very small proportion of $\mathrm{TH}$ is phosphorylated (Haycock et al., 1998; Salvatore et al., 2001; Bobrovskaya et al., 2004), immunostaining for the phosphoryated $\mathrm{TH}$ (THp) forms was significantly lower than for native $\mathrm{TH}$, and was restricted to discrete striatal regions. Thus, immunoreactivity for THp31 was virtually undetected in our material (data not shown), and for THp19 and THp40 was confined to the olfactory tubercle (OT), the accumbens shell and small patches throughout the ventral and dorsal striatum (Figures 2B,C). The similarity in shape and distribution between THp patches and striosomes (see Graybiel and Ragsdale, 1978) prompted us to perform double labeling for
THp19 or THp40 and mu-opioid receptor (a marker of striosomes; Desban et al., 1993). However, no colocalization between both markers was found (Figure 2D), indicating that THp-rich patches preferentially localize in the matriceal compartment of the striatum. Interestingly, all the patches surround vascular profiles. The combination of immunolabeling for THp (Ser19 or Ser40) and two vessel markers, the endothelial form of nitric oxide synthase (eNOS, Figure 2E) and vimentin (Figure 2F), confirmed that THp-rich patches form dense terminal plexuses around striatal vessels, even penetrating their external layers (Figures 2J,K arrows). The presence of THp perivascular plexuses was also explored in monkeys. As shown in Figures $2 \mathrm{G}-\mathbf{I}$, small and large vessels in the ventral and dorsal striatum of monkeys were also surrounded by dense plexuses of THp terminals. Furthermore, double labeling for THp (Ser19 or Ser40) and GFAP in rats showed that THp-terminals are in close apposition to perivascular astroglial processes (Figures $\mathbf{2} \mathbf{L}, \mathbf{M}$ arrows).

As previously reported (González-Hernández et al., 2004), immunostaining for DAT showed a homogeneous field of welldefined striatal terminals (Figure 3A). A low magnification view of double labeling for DAT and THp (Ser 19 and Ser 40 ) in rats showed co-localization in practically all perivascular plexuses (Figures $3 \mathrm{~A}-\mathrm{C}$ ). The count of double labeled terminals at higher magnification revealed that within $100 \mu \mathrm{m}$ from striatal vessels, virtually $100 \%(98.4 \pm 0.9 \%)$ of positive THp19 terminals were immunoreactive for DAT, and $97.7 \pm$ $1.1 \%$ of DAT terminals were also immunoreactive for THp19 (Figures 3D-F). Furthermore, $97.4 \pm 0.8 \%$ of THp40 terminals were immunoreactive for DAT, but THp40 immunoreactivity was not detected in $12.8 \pm 1.3 \%$ of DAT terminals. These findings confirm the dopaminergic nature of THp plexuses and that both serine residues are phosphorylated in most perivascular terminals. Further supporting the midbrain dopaminergic origin of the THp-rich innervation of striatal vessels, BDA-positive axons and perivascular terminals were immunoreactive for both DAT (Figures 3G-I) and THp19 (Figures 3J-L) or THp40 (Figures 3M-O). Moreover, striatal THp perivascular plexuses disappeared after midbrain dopaminergic lesion in parallel with the loss of midbrain DA-cells (6-OHDA, Figure 4). In sum, our results indicate that striatal vessels receive midbrain dopaminergic inputs that form dense perivascular plexuses of THp-rich terminals.

\section{DISCUSSION}

The cerebral blood flow is controlled by two major neurovascular systems. One of them, known as the extrinsic system, consists of fibers from sympathetic and parasympathetic extracranial ganglia and the sensory trigeminal ganglion that release different vasoactive substances on the wall of extracerebral arteries and pial arterioles for the regulation of the global cerebral perfusion (Drake and Iadecola, 2007; Willie et al., 2014). The other one, known as the intrinsic system, consists of fibers arising from different neurons in the brain which project directly to parenchymal microvessels or astroglial end-feet adjoining them for the regulation of highly localized changes in blood flow coupled with regional synaptic activity (Iadecola, 2004; Hamel, 2006; Drake and Iadecola, 2007; Attwell et al., 2010; Lecrux and 


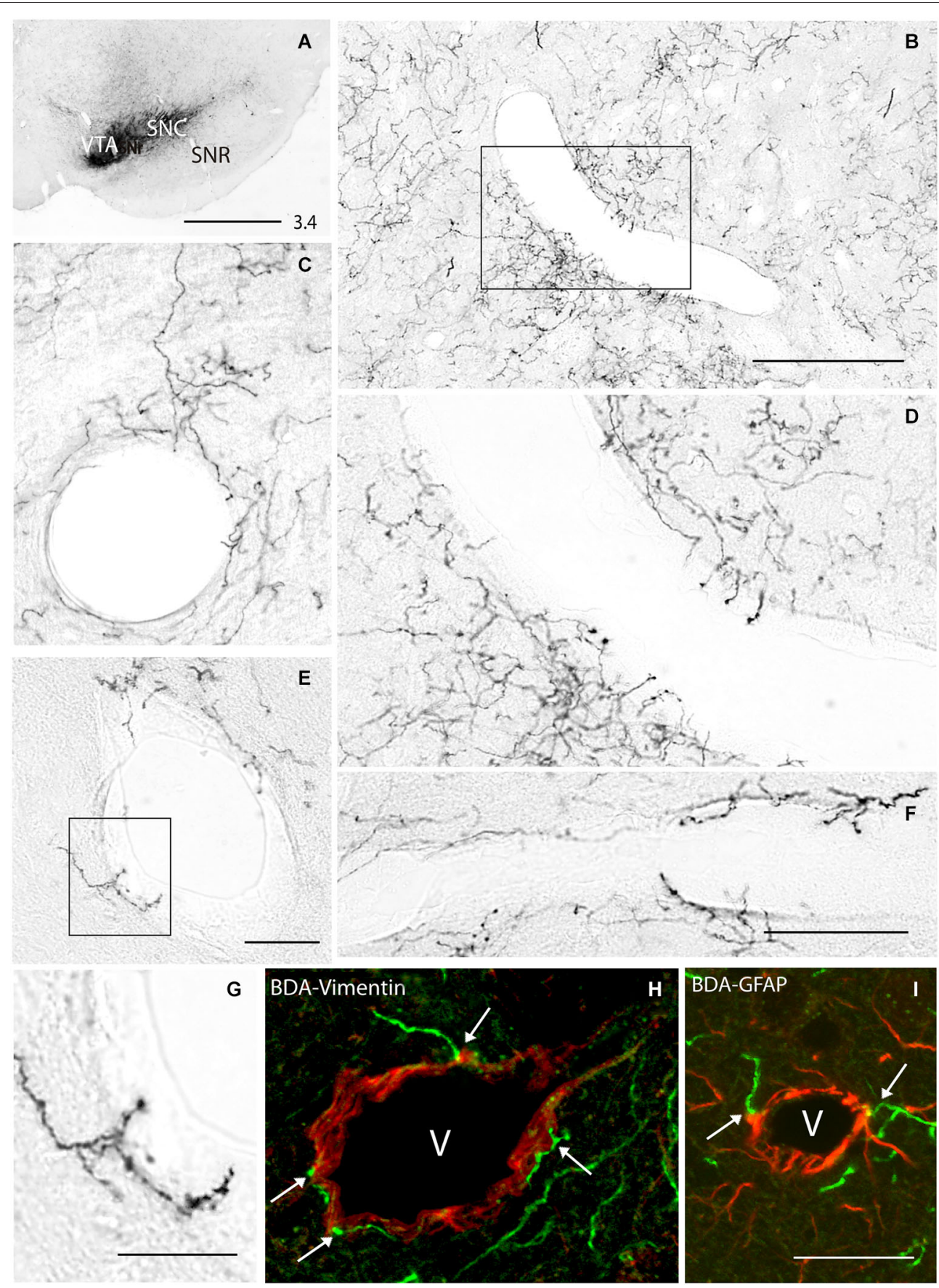

FIGURE 1 | (A) Biotinylated dextran amine (BDA) injection in the substantia nigra pars compacta (SNC) and ventral tegmental area (VTA) of the rat. (B-G) BDA-positive fibers emitting terminals in close apposition to striatal vessels, some of them in the form of individual axons (C,E, G), and others forming sparse plexuses (B,D,F). (H,I) Double labeling for BDA and vimentin (H) or GFAP (I) showing terminals abutted on striatal vessels and surrounding astrocytes (arrows in $\mathbf{H}$ and $\mathbf{I}$ ). (D, $\mathbf{G})$ Boxed areas in $(\mathbf{B})$ and $(\mathbf{E})$ respectively. The number at the bottom right in $(\mathbf{A})$ indicates the distance from the interaural axis in millimeters. SNR, substantia nigra pars reticulata. $\mathrm{V}_{\text {, }}$ vessel lumen. Bar in (A), $1 \mathrm{~mm}$; in B, $400 \mu \mathrm{m}$; in (F) (for D and F), 150 $\mu \mathrm{m}$; in (G), $100 \mu \mathrm{m}$; in (I) (for $\mathbf{H}$ and I), $100 \mu \mathrm{m}$.
Hamel, 2011). This system, preferentially studied in the cerebral cortex, involves local GABAergic, peptidergic and glutamatergic collaterals, as well as cholinergic, noradrenergic and serotoninergic ascending inputs (Attwell and Iadecola, 2002; Cauli et al.,
2004; Iadecola, 2004; Lecrux and Hamel, 2011). The use of anterograde tracers, specific neurotoxins and electron microscopy shows that these projections come from the nucleus basalis, locus coeruleus, and raphe nuclei respectively (Reinhard et al., 1979; 

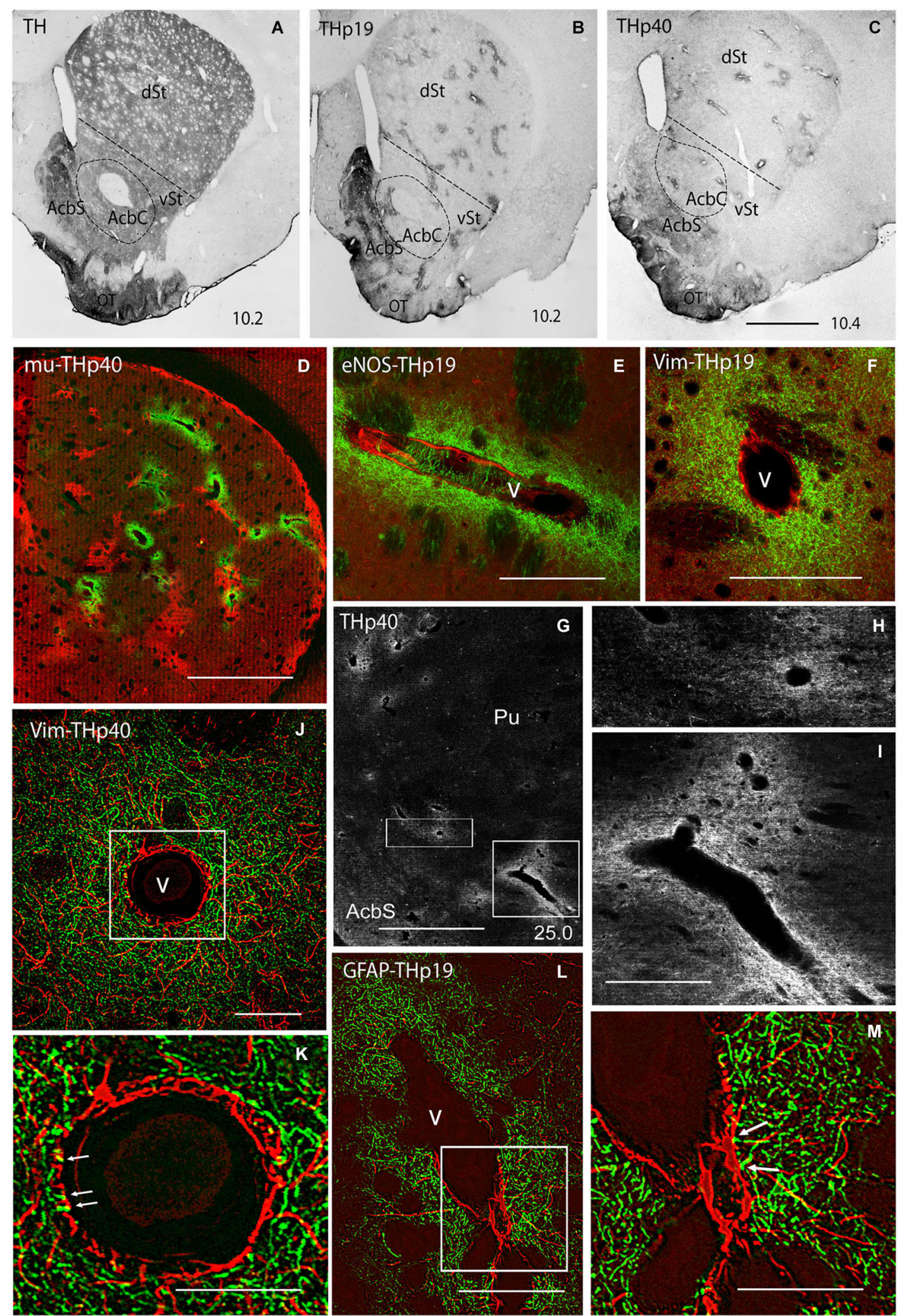

FIGURE 2 | (A-C) Immunostaining for the non-phosphorylated (TH; A) and phosphorylated forms of tyrosine hydroxylase at Ser19 (THp19; B) and Ser40 (THp40; C) in rat striatum. We note that in contrast to the homogeneous labeling for $\mathrm{TH}$ (A), THp19 and THp40 immunostaining is restricted to the olfactory tubercle (OT), accumbens shell (AcbS), and discrete regions throughout the ventral (vSt) and dorsal (dSt) striatum. (D) Double labeling for mu-opioid receptor (mu, a marker of striosomes; red) and THp40 (green) showing that THp40 is not localized in striosomes. (E,F) Double labeling for endothelial nitric oxide synthase (eNOS; E, red) or

(Continued) 


\section{FIGURE 2 | Continued}

vimentin (Vim; $\mathbf{F}$, red) and THp19 (green) showing dense plexuses of THp19-positive terminals surrounding longitudinal- (E) and transversally (F) cut vessels. (G) Immunostaining for THp40 in the monkey striatum showing intense immunoreactivity in perivascular terminals. (H,I) Boxed areas in (G). (J) Double labeling for vimentin (Vim; red) and THp40 (green) in rat striatum subjected to deconvolution processing. (K) Boxed area in (J) showing THp40 terminals in close apposition to or penetrating (arrows) the vessel wall (arrows). (L) Double labeling for GFAP (red) and THp19 (green) in rat striatum subjected to deconvolution processing. (M) Boxed area in (L) showing THp19 terminals touching astroglial processes (arrows). Doted line in (A-C) indicates the putatitve border between vSt and dSt. The number at the bottom right in $\mathbf{A} \mathbf{A}-\mathbf{C}, \mathbf{G})$ indicates the distance from the interaural axis in millimeters. AcbC, accumbens core; Pu, putamen; $v$, vessel lumen. Bar in (C) (for $\mathbf{A}-\mathbf{C}), 450 \mu \mathrm{m}$; in (D), $200 \mu \mathrm{m}$; in (E), $75 \mu \mathrm{m}$; in (F), $75 \mu \mathrm{m}$; in (G), $3 \mathrm{~mm}$; in (I) (for $\mathbf{H}$ and I), $1 \mathrm{~mm}$; in (J), $50 \mu \mathrm{m}$; in (K), $20 \mu \mathrm{m}$; in (L), $50 \mu \mathrm{m}$; in (M), $20 \mu \mathrm{m}$.

Vaucher and Hamel, 1995; Cohen et al., 1997), and that their endings make contact with both the components of the neurovascular unit, the vessel wall and surrounding astrocytes (Vaucher and Hamel, 1995; Cohen et al., 1997). Dopaminergic neurotransmission has also been implicated in both neurovascular regulatory systems. Firstly, through in vitro and in situ studies showing that DA and its analogs promote vasoactive changes in convexity cerebral arteries and pial arterioles (Toda, 1976; Edvinsson et al., 1978a,b, 1985; Forster et al., 1983), and more recently, from the evidence of dopaminergic contacts in capillaries and perivascular astrocytes (Krimer et al., 1998), and the induction of hemodynamic changes in discrete brain regions by dopaminergic drugs (Marota et al., 2000; Choi et al., 2006; Sander et al., 2013) and their inhibition after dopaminergic lesion (Chen et al., 1997; Nguyen et al., 2000; Jenkins et al., 2004). However, the anatomical origin of dopaminergic inputs to intracerebral vessels has not been elucidated. The results here, based on the injection of an anterograde neuronal tracer, combination of vessel, astrocyte and dopaminergic markers, confocal microscopy and dopaminergic lesion, indicate that striatal vessels receive inputs from midbrain dopaminergic neurons. We are aware that only electron microscopy provides high enough magnification to properly identify synaptic structures. Nevertheless, high-resolution confocal laser scanning followed by adequate post-adquisition computer processing markedly reduces the resolution gap between optical and electron microscopies (Sibarita, 2005; Salin et al., 2008). Thus, one can suggest that, similar to dopaminergic and nondopaminergic inputs to cortical vessels (Vaucher and Hamel, 1995; Cohen et al., 1997; Krimer et al., 1998), ascending dopaminergic terminals make contact with both components of the neurovascular unit in the rat striatum. In any case, we know that besides the synaptic transmission which requires interneuronal contacts, neurotransmitters and neuromodulators, including DA, can signal through extrasynaptic receptors after flowing short or long distances in the extracellular space. Interestingly, electron microscopy studies show that $50 \%$ of $\mathrm{DA} \mathrm{D}_{1}$ and $\mathrm{D}_{2}$ receptors in the rat striatum are extrasynaptic (Yung et al., 1995), suggesting that most dopaminergic afferents can operate via both synaptic and extrasynaptic receptors. According to Fuxe et al. (2012), this sort of intercellular communication called volume transmission (Agnati et al., 1986), is the main communication pathway in neuroglial and neurovascular interactions. The evidence of $D A D_{1}$ and $D_{2}$ receptors in endothelial and astroglial cells (Bacic et al., 1991; Bal et al., 1994; Zanassi et al., 1999; Choi et al., 2006), together with the time (0-2 s) of vascular response to DA release in the striatum (Knutson and Gibbs, 2007) agree with the suggestion of Fuxe et al. (2012), and therefore the evidence of terminal contacts becomes less relevant.

Our results further show that dopaminergic axons innervating striatal vessels form dense perivascular plexuses immunoreactive for phosphorylated TH at Ser19 and Ser40. We know that phosphorylation is the primary mechanism responsible for shortterm activation of $\mathrm{TH}$, the rate-limiting enzyme of catecholamine synthesis, and that TH may be phosphorylated by different protein kinases at four serine residues $(8,19,31$ and 40) near $\mathrm{N}$ terminus (Haycock and Wakade, 1992; Dunkley et al., 2004; Nakashima et al., 2009). However, under basal conditions no more than $5-7 \%$ TH is phosphorylated, and a substantial proportion of $\mathrm{TH}$ becomes phosphorylated only after protein kinase activating stimuli (Pocotte et al., 1986; Haycock, 1993; Haycock et al., 1998; Salvatore et al., 2001; Bobrovskaya et al., 2004). Consistent with these data, $\mathrm{Xu}$ et al. (1998) reported reduced immunoreactivity for phosphorylated $\mathrm{TH}$ in comparison with that for the native form, with phosphorylation being restricted to Ser19 and localized in the accumbens nucleus and the OT. Our results confirm these findings but enlarging them show that $\mathrm{TH}$ is also constitutively phosphorylated in dopaminergic terminals encircling vessels throughout the ventral and dorsal striatum. In addition, as demonstrated in rats and monkeys, phosphorylation also involves Ser40 residue.

The functional meaning and advantages of having multiple phosphorylatable positions have not been yet elucidated (for review see Daubner et al., 2011), but there is a general consensus in that Ser40 is critical for TH activity. Thus, while no activation mechanisms and functional consequences have been found for phosphorylation at Ser8 and phosphorylation at Ser19 and Ser31 promote no more than 2 -fold increases in $\mathrm{TH}$ activity, phosphorylation at Ser40 results in a 300 -fold decrease in $\mathrm{TH}$ affinity for catecholamines with a 20 -fold increase in TH activity (Bevilaqua et al., 2001; Bobrovskaya et al., 2004; Dunkley et al., 2004; Daubner et al., 2011). In addition, although Ser19 phosphorylation "per se" does not significantly affect TH activity, it modifies TH structure thereby accelerating its phosphorylation at Ser40 (Bevilaqua et al., 2001). Consequently, one can assume that dopaminergic terminals with TH phosphorylated at Ser19 and Ser40 are responsible for the basal $\mathrm{TH}$ activity in the striatum, and that their DA content ready to be released is higher than in those without phosphorylated $\mathrm{TH}$.

Studies about the striatal processing in associative learning indicate that the activity of striatal neurons in each trial is preceded by adaptive local hemodynamic changes which are mediated by DA (Knutson and Gibbs, 2007; Peterson and Seger, 2013). Midbrain dopaminergic neurons fire in response to reward-predicting cues leading to an increase in extracellular DA levels in the striatum (Roitman et al., 2004). Changes in extracellular DA levels are paralleled by an increase in the regional 

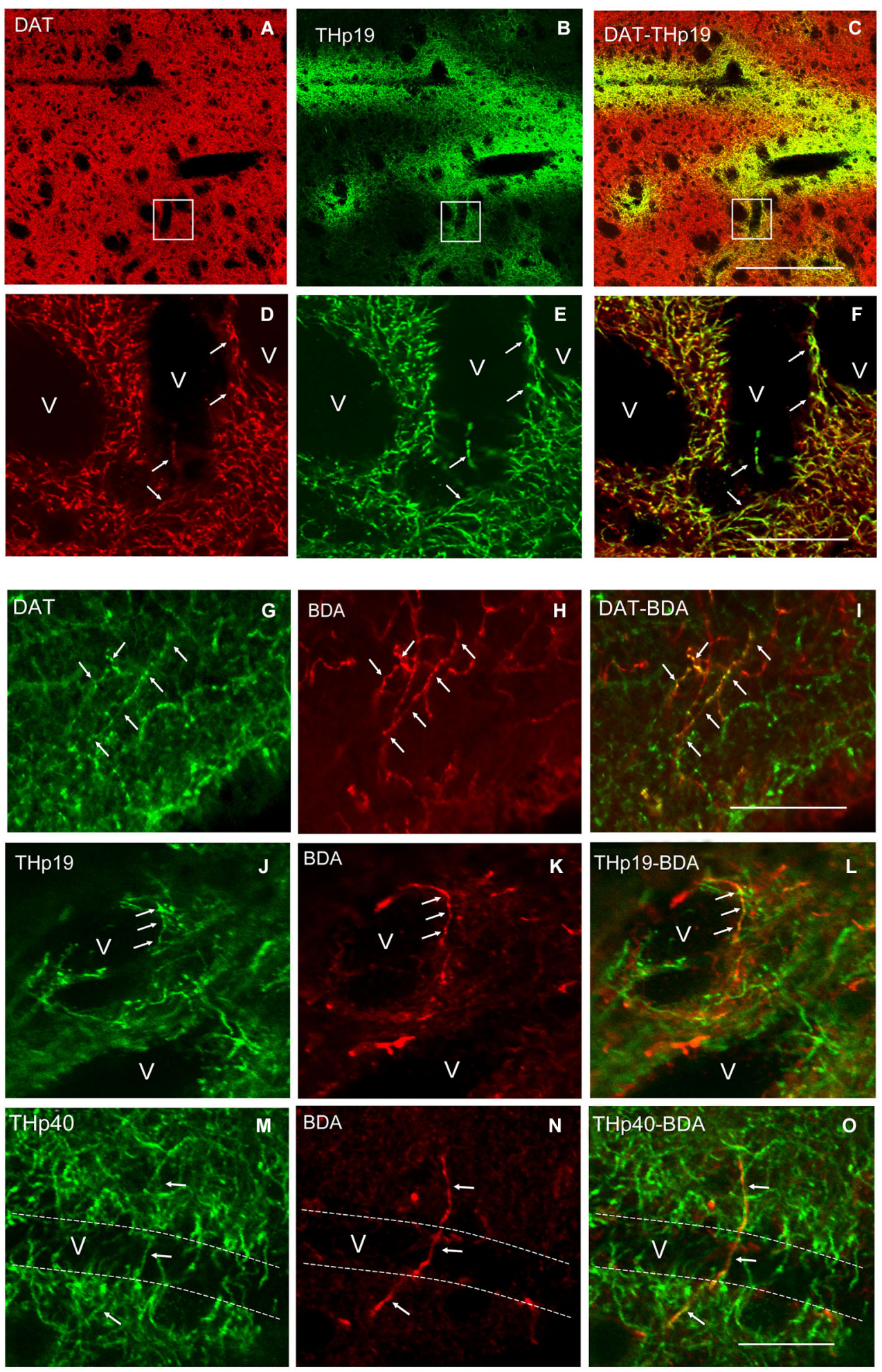

FIGURE 3 | (A-C) A low magnification view of double labeling for the dopamine transporter (DAT) and THp19 in the dorsal striatum of the rat. (D-F) Boxed areas in (A), (B) and (C) respectively showing that all perivascular THp19 fibers and terminals are dopaminergic (DAT-immunoreactive). (G-O) Double labeling for DAT, THp19 or THp40 and
BDA showing that BDA-positive ascending axons $\mathbf{( H )}$ and perivascular terminals $(\mathbf{K}, \mathbf{N})$ are immunoreactive for DAT $(\mathbf{G}-\mathbf{I})$, THp19 (J-L) and THp40 (M-O). $\mathbf{v}$, vessel lumen. Dotted lines in (M-O) indicate the probable localization of the vessel wall. Bar in (C) (for $\mathbf{A}-\mathbf{C}$ ), $130 \mu \mathrm{m}$; in (F) (for D-F), $40 \mu \mathrm{m}$; in (I) (for $\mathbf{G}-\mathbf{I}$ ), $40 \mu \mathrm{m}$; in (O) (for $\mathbf{J}-\mathbf{O}$ ), $40 \mu \mathrm{m}$. 


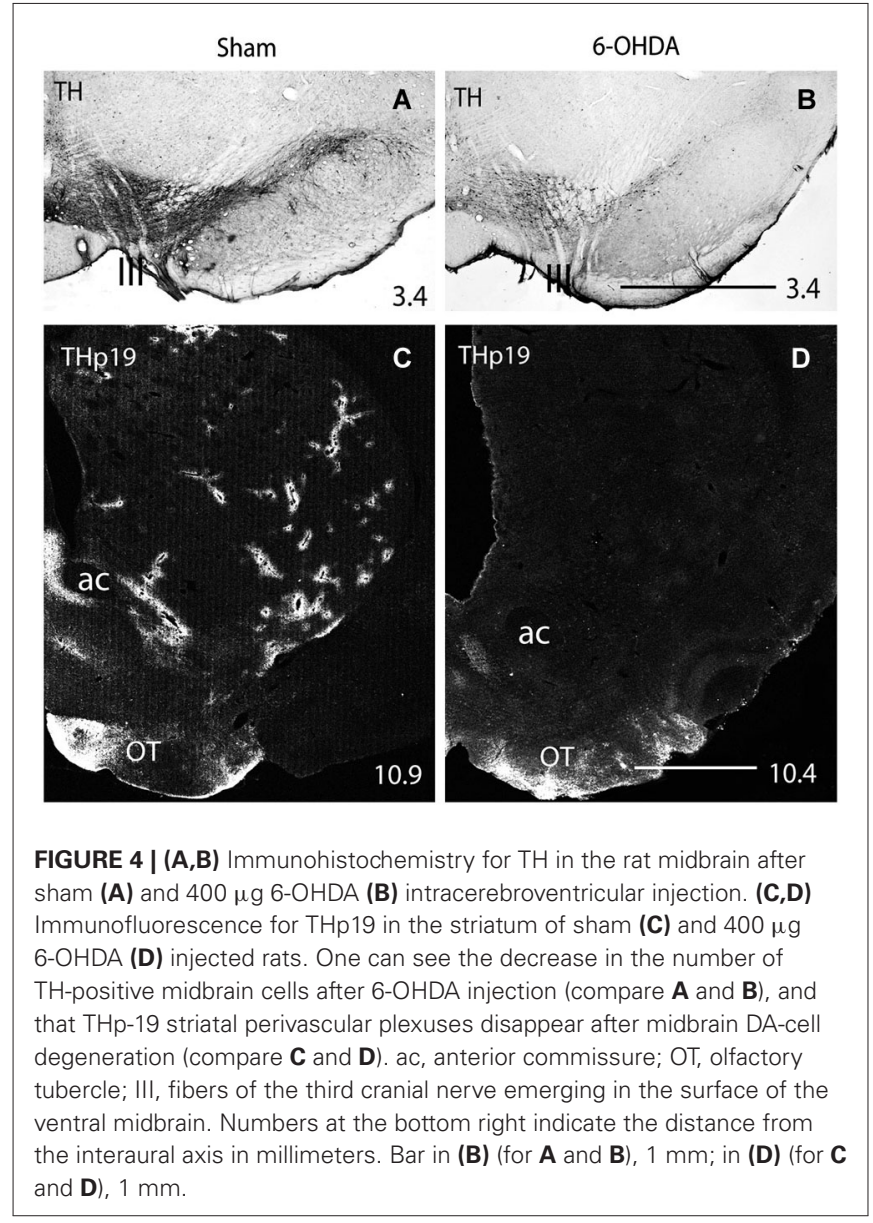

cerebral blood flow that is inhibited by dopaminergic lesion (Chen et al., 1997; Nguyen et al., 2000; Mandeville et al., 2001; Jenkins et al., 2004). Consequently, the prior release of DA in discrete striatal regions is nowadays considered a key factor in the regulation of blood flow depending on local metabolic demands. It is notable that dopaminergic signaling has also been involved in anticipatory hemodynamic changes in the cerebral cortex (Tan, 2009). However, in the light of our and previous results, this should be quite different to that in the striatum. In contrast to the conspicuous perivascular plexuses involving small to large vessels from the OT to the dorsal striatum, dopaminergic terminals are sparse and restricted to small vessels in the cerebral cortex (Krimer et al., 1998). Furthermore, as shown in rats and monkeys, $\mathrm{TH}$ is not constitutively phosphorylated in vessel terminals in the cerebral cortex. The restricted localization of phosphorylated $\mathrm{TH}$ in dopaminergic terminals of striatal vessels suggests that it is related to specific aspects of striatal processing. As shown in Figure 5, a striatal neurovascular unit and the neighboring neuropil probably receive projections from the same midbrain neuron. Consequently, dopaminergic inputs should reach both compartments at the same time. However, the fact that dopaminergic terminals in perivascular plexuses contain phosphorylated $\mathrm{TH}$ facilitates that the release of DA onto vessels precedes that onto the neighboring neuropil, and then, that DA vascular actions precede DA neuronal (non phosphorylated $\mathrm{TH}$ ) actions.

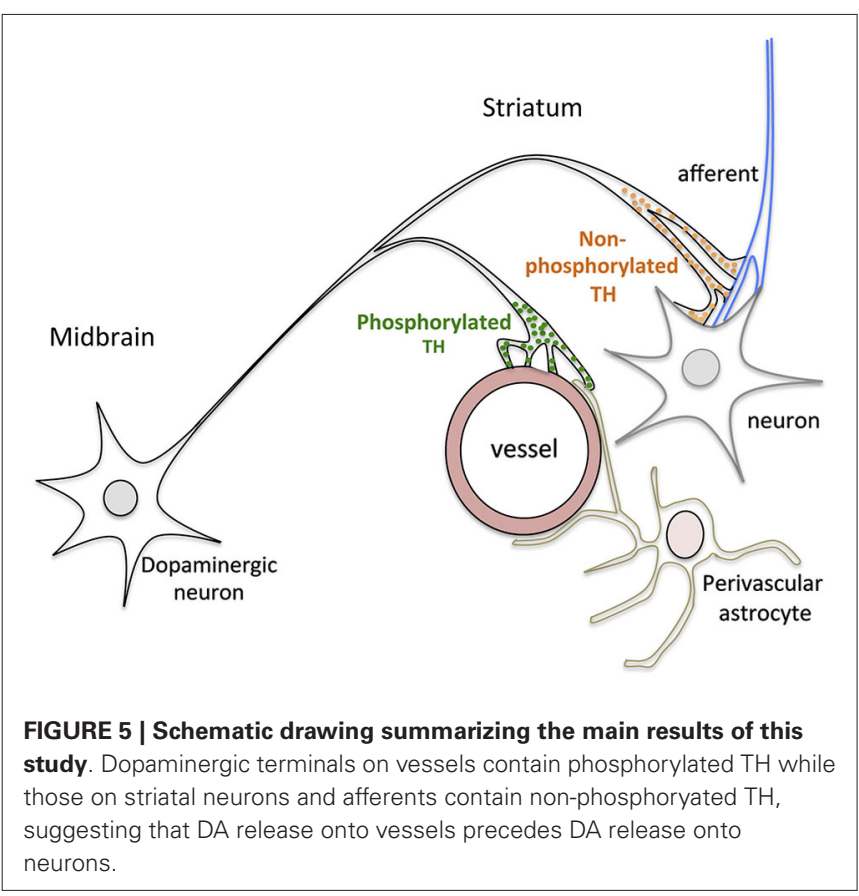

We know that the striatum receives the most intense dopaminergic input in the brain, and that DA actions are exerted through different DA-receptors in striatal neurons and cortical and subcortical afferents arriving to different striatal regions (Obeso et al., 2008). In addition, the loss of DA signaling in striatal circuits is considered to cause motor symptoms as well as less noticeable cognitive and psychiatric manifestations of PD (Rodriguez-Oroz et al., 2009). However, as our results show, an important contingent of dopaminergic axons reaches striatal vessels rather than neurons, suggesting that these axons can play a role in the pathophysiology of PD. Beyond the concept of vascular parkinsonism (Kalra et al., 2010), the relationship between genuine PD and cerebral perfusion has mostly been focused on whether vascular co-morbidities can aggravate motor symptoms as a result of additive effects of two independent disorders (Kotagal et al., 2014), or the possibility that vascular aspects can act synergistically with other factors contributing to dopaminergic cell degeneration. In support of the latter, morphological changes have been found in nigral vessels of PD patients and animal models of PD (Faucheux et al., 1999; Barcia et al., 2005), and the density of nigral microvessels has also been found to be low in aged rats (VillarCheda et al., 2009). In addition, recent studies by RodriguezPerez et al. (2013) reveal that chronic brain hypoperfusion can "per se" induce dopaminergic cell degeneration and exacerbate the degeneration promoted by intrastriatal injection of 6-OHDA. Our findings suggest that independently of the vascular involvement in dopaminergic degeneration, the striatal blood flow can also be substantially affected as a result of dopaminergic cell degeneration. Consequently, the supply of oxygen and nutrients coupled to hemodynamic changes preceding neuronal activity might also be altered in the striatum, contributing as an additional factor in the pathophysiology of PD from its first stages. Interestingly, although the relationship between neuronal activity and oxygen 
consumption is the basis of functional imaging technologies widely used in the diagnosis of PD (Mahlknecht et al., 2010), nowadays blood flow is considered to merely serve as a metabolic support in this paradigm (Moore and Cao, 2008). Moreover, changes in striatal perfusion in the course of PD are still a matter of controversy. While some authors report an increase of the regional blood flow (Wolfson et al., 1985; Feigin et al., 2002; Hsu et al., 2007), others find no changes (Bissessur et al., 1997) or a decrease (Markus et al., 1994; Van Laere et al., 2004). These discrepancies reflect differences in data processing and the clinical profile of patients studied. In this respect, Imon et al. (1999) found a decrease in striatal blood flow at early stages of PD, and an increase at later stages, suggesting that changes can depend on the disease stage. Bearing in mind the relative weight of the "vascular component" in the mesostriatal pathway, it is possible that hemodynamic changes may be detected and monitored from the early stages of PD with the systematic use of functional imaging.

\section{ACKNOWLEDGMENTS}

This work was supported by Ministerio de Ciencia e Innovación (grant no. BFU2010-21130). Javier Castro-Hernández was supported by a predoctoral fellowship from Fundación Canaria de Invetigación y Salud, Josmar Salas-Hernández by a grant from the Gobierno Autónomo de Canarias, and Pedro Barroso-Chinea by a grant from The Excellence Tri-continental Atlantic Campus (University of La Laguna) and the IMBRAIN project (FP7-REGPOT2012-CT2012-31637-IMBRAIN), funded by the 7th Framework Program (Capacities).

\section{REFERENCES}

Agnati, L. F., Fuxe, K., Zoli, M., Ozini, I., Toffano, G., and Ferraguti, F. (1986). A correlation analysis of the regional distribution of central enkephalin and betaendorphin immunoreactive terminals and of opiate receptors in adult and old male rats. Evidence for the existence of two main types of communication in the central nervous system: the volume transmission and the wiring transmission. Acta Physiol. Scand. 128, 201-207. doi: 10.1111/j.1748-1716.1986.tb07967.x

Attwell, D., Buchan, A. M., Charpak, S., Lauritzen, M., MacVicar, B. A., and Newman, E. S. (2010). Glial and neuronal control of brain blood flow. Nature 468, 232-243. doi: 10.1038/nature09613

Attwell, D., and Iadecola, C. (2002). The neuronal basis of functional brain imaging signals. Trends Neurosci. 25, 621-625. doi: 10.1016/s0166-2236(02)02264-6

Bacic, F., Uematsu, S., McCarron, R. M., and Spatz, M. (1991). Dopaminergic receptors linked to adenylate cyclase in human cerebromicrovascular endothelium. J. Neurochem. 57, 1774-1780. doi: 10.1111/j.1471-4159.1991.tb06380.x

Bal, A., Bachelot, T., Savasta, M., Manier, M., Verna, J. M., Benabid, A. L., et al. (1994). Evidence for dopamine D2 receptor mRNA expression by striatal astrocytes in culture: in situ hybridization and polymerase chain reaction studies. Brain Res. Mol. Brain Res. 23, 204-212. doi: 10.1016/0169-328x(94)90227-5

Barcia, C., Bautista, V., Sanchez-Bahillo, A., Fernandez-Villalba, E., Faucheux, B., Poza y Poza, M., et al. (2005). Changes in vascularization in substantia nigra pars compacta of monkeys rendered parkinsonian. J. Neural Transm. 112, 12371248. doi: 10.1007/s00702-004-0256-2

Bevilaqua, L. R. M., Graham, M. E., Dunkley, P. R., von Nagy-Felsobuki, E. I., and Dickson, P. W. (2001). Phosphorylation of ser(19) alters the conformation of tyrosine hydroxylase to increase the rateod phosphorylation of ser(40). J. Biol. Chem. 276, 40411-40416. doi: 10.1074/jbc.m105280200

Bissessur, S., Tissingh, G., Wolters, E. C., and Scheltens, P. (1997). rCFB SPECT in Parkinson's disease patients with mental dysfuction. J. Neural Transm. Suppl. 50, 25-30. doi: 10.1007/978-3-7091-6842-4_3

Bobrovskaya, L., Dunkley, P. R., and Dickson, P. W. (2004). Phosphorylation of ser19 increases both ser40 phosphorylation and enzyme activity of tyrosine hydroxylase in intact cells. J. Neurochem. 90, 857-864. doi: 10.1111/j.1471-4159. 2004.02550.x

Breiter, H. C., Gollub, R. L., Weisskoff, R. M., Kennedy, D. N., Makris, N., Berke, J. D., et al. (1997). Acute effects of cocaine on human brain activity and emotion. Neuron 19, 591-611. doi: 10.1016/s0896-6273(00)80374-8

Cauli, B., Tong, X. K., Rancillac, A., Serluca, N., Lambolez, B., Rossier, J., et al. (2004). Cortical GABA interneurons in neurovascular coupling: relays for subcortical vasoactive pathways. J. Neurosci. 24, 8940-8949. doi: 10.1523/jneurosci. 3065-04.2004

Chen, Y. C., Garpern, W. R., Brownell, A. L., Matthews, R. T., Bogdanov, M., Isacson, O., et al. (1997). Detection of dopaminergic neurotransmitter activity using pharmacologic MRI: correlation with PET microdyalisis and behavioural data. Mag. Res. Med. 38, 389-398. doi: 10.1002/mrm.1910380306

Choi, J.-K., Chen, Y. I., Hamel, E., and Jenkins, B. G. (2006). Brain hemodynamic changes mediated by dopamine receptors: role of the cerebral microvasculature in dopamine-mediated neurovascular coupling. Neuroimage 30, 700-712. doi: 10.1016/j.neuroimage.2005.10.029

Cohen, Z., Molinatti, G., and Hamel, E. (1997). Astroglial and vascular interactions of noradrenaline terminals in the rat cerebral cortex. J. Cereb. Blood Flow Metab. 17, 894-904. doi: 10.1097/00004647-199708000-00008

Daubner, S. C., Le, T., and Wang, S. (2011). Tyrosine hydroxylase and regulation of dopamine synthesis. Arch. Biochem. Biophys. 508, 1-12. doi: 10.1016/j.abb.2010. 12.017

Desban, M., Kemel, M. L., Glowinski, J., and Gauchy, C. (1993). Spatial organization of patch and matrix compartments in the rat striatum. Neuroscience 57, 661-671. doi: 10.1016/0306-4522(93)90013-6

Drake, C. T., and Iadecola, C. (2007). The role of neuronal signalling in controlling cerebral blood flood. Brain Lang. 102, 141-152. doi: 10.1016/j.bandl.2006.08. 002

Dunkley, P. R., Bobrovskaya, L., Graham, M. E., von Nagy-Felsobuki, E. I., and Dickson, P. W. (2004). Tyrosine hydroxylase phosphorylation: regulation and consequences. J. Neurochem. 91, 1025-1043. doi: 10.1111/j.1471-4159.2004. 02797.x

Edvinsson, L., Hardebo, J.-E., McCulloch, J., and Owman, C. (1978a). Effects of dopaminergic agonists on isolated cerebral blood vessels. Acta Physiol. Scand. 104, 349-359. doi: 10.1111/j.1748-1716.1978.tb06286.x

Edvinsson, L., Hardebo, J.-E., McCulloch, J., and Owman, C. (1978b). "Vasomotor response of the cerebral blood vessels to dopamine and dopaminergic agonists," in Advances in Neurology. Vol. 20: Pathology of Cerebrospinal Microcirculation, eds J. Cervós-Navarro, E. Betz, G. Ebhardt, R. Ferszt and R. Wüllenweber (New York: Raven Press), 85-96.

Edvinsson, L., McCulloch, J., and Shankey, J. (1985). Vasomotor response of cerebral arterioles in situ to putative dopamine receptor agonists. Br. J. Pharmacol. 85, 403-410. doi: 10.1111/j.1476-5381.1985.tb08875.x

Faucheux, B. A., Bonnet, A. M., Agid, Y., and Hirsch, E. C. (1999). Blood vessels change in the mesencephalon of patients with Parkinson's disease. Lancet 353, 981-982. doi: 10.1016/s0140-6736(99)00641-8

Feigin, A., Antonini, A., Fukuda, M., De Notaris, R., Benti, R., Pezzoli, G., et al. (2002). Tc-99m ethylene cysteinate dimer SPECT in the differential diagnosis of parkinsonim. Mov. Disord. 17, 1265-1270. doi: 10.1002/mds.10270

Forster, C., Drew, G. M., Hilditch, A., and Whalley, E. T. (1983). Dopamine receptors in human basilar arteries. Eur. J. Pharmacol. 87, 227-235. doi: 10. 1016/0014-2999(83)90332-1

Fuxe, K., Borroto-Escuela, D. O., Romero-Fernandez, W., Diaz-Cabiale, Z., Rivera, A., Ferraro, L., et al. (2012). Extrasynaptic neurotransmission in the modulation of brain function. Focus on the striatal neuronal-glial networks. Front. Physiol. 3:136. doi: 10.3389/fphys.2012.00136

Gerfen, C. R., Herkenham, M., and Tribault, J. (1987). The neostriatal mosaic: II. Path- and matrix-directed mesostriatal dopaminergic and nondopaminergic systems. J. Neurosci. 7, 3915-3934. Available online at: http://www.jneurosci.org/content/7/12/3915.long.

González-Hernández, T., Barroso-Chinea, P., De La Cruz-Muros, I., Perez-Delgado, M. M., and Rodriguez, M. (2004). Expression of dopamine and vesicular monoamine transporters and differential vulnerability of mesostriatal dopaminergic neurons. J. Comp. Neurol. 479, 198-215. doi: 10.1002/cne.20323

Graybiel, A. M., and Ragsdale, C. W. (1978). Histochemically distinct compartments in the striatum of human, monkey and cat demonstrated by acetylcholinesterase staining. Proc. Natl. Acad. Sci. U S A 75, 5723-5726. doi: 10. 1073/pnas.75.11.5723 
Hamel, E. (2006). Perivascular nerves and the regulation of cerebrovascular tone. J. Appl. Physiol. 100, 1059-1064. doi: 10.1152/japplphysiol.00954.2005

Haycock, J. W. (1993). Multiple signalling pathways in bovine chromaffin cells regulate tyrosine hydroxylase phosphorylation at Ser19, Ser31 and Ser40. Neurochem. Res. 18, 15-26. doi: 10.1007/bf00966919

Haycock, J. W., Lew, J. Y., Garcia-Espana, A., Lee, K. Y., Harada, K., Meller, E., et al. (1998). Role of serine-19 phosphorylation in regulating tyrosine hydroxylase studied with site- and phosphospecific antibodies and site-directed mutagenesis. J. Neurochem. 71, 1670-1675. doi: 10.1046/j.1471-4159.1998. 71041670.x

Haycock, J. W., and Wakade, A. R. (1992). Activation and multiple-site phosphorylation of tyrosine hydroxylase in perfused rat adrenal glands. J. Neurochem. 58, 57-64. doi: 10.1111/j.1471-4159.1992.tb09276.x

Hsu, J.-L., Jung, T.-P., Hsu, C.-Y., Hsu, W.-C., Chen, Y.-K., Duann, J.-R., et al. (2007). Regional CBF changes in Parkinson's disease: a correlation with motor dysfunction. Eur. J. Nucl. Med. Mol. Imaging 34, 1458-1466. doi: 10. 1007/s00259-006-0360-7

Iadecola, C. (2004). Neurovascular regulation in the normal brain and in Alzheimer's disease. Nat. Rev. Neurosci. 5, 347-360. doi: 10.1038/nrn1387

Imon, Y., Matsuda, H., Ogawa, M., Kogure, D., and Sunohara, N. (1999). SPECT image analysis using statistical parametric mapping in patients with Parkinson's disease. J. Nucl. Med. 40, 1583-1589. Available online at: http://jnm.snmjournals.org/content/40/10/1583

Ingvar, M., Lindvall, O., Folbergrová, J., and Siesjö, B. K. (1983). Influence of lesions of the noradrenergic locus coeruleus system on the cerebral metabolic response to bicuculline-induced seizures. Brain Res. 264, 225-231. doi: 10.1016/00068993(83) $90820-\mathrm{x}$

Jenkins, B. G., Sanchez-Penaute, R., Brownell, A. L., Chen, Y. C., and Isacson, O. (2004). Mapping dopamine fuction in primates using pharmacological magnetic resonance imaging. J. Neurosci. 24, 9553-9560. doi: 10.1523/jneurosci. 1558-04.2004

Joel, D., and Weiner, I. (2000). The connections of the dopaminergic system with the striatum in rats and primates: an analysis with respect to the functional and compartmental organization of the striatum. Neuroscience 96, 451-474. doi: 10. 1016/S0306-4522(99)00575-8

Kalra, S., Grosset, D. G., and Benamer, H. T. (2010). Differentiating vascular parkinsonism from idiopathic Parkinson's disease: a systematic review. Mov. Disord. 25, 149-156. doi: 10.1002/mds.22937

Knutson, B., and Gibbs, S. E. B. (2007). Linking nucleus accumbens dopamine and blood oxygenation. Psychopharmacology (Berl) 191, 813-822. doi: 10. 1007/s00213-006-0686-7

Kotagal, V., Albin, R. L., Müller, M. L., Koeppe, R. A., Frey, K. A., and Bohnen, N. I. (2014). Modifiable cardiovascular risk factors and axial motor impairments in Parkinson's disease. Neurology 82, 1514-1520. doi: 10.1212/wnl. 0000000000000356

Krimer, L. S., Muly, E. C., Williams, G. V., and Goldman-Rakic, P. S. (1998). Dopaminergic regulation of cerebral cortical microcirculation. Nat. Neurosci. 1, 286-289. doi: 10.1038/1099

Lanciego, J. L., and Wouterlood, F. G. (2006). "Multiple neuroanatomical tracttracing: approaches for multiple tract-tracing," in Neuroanatomical TractTracing 3. Molecules, Neurons and Systems, eds L. Zaborszky, F. G. Wouterlood and J. L. Lanciego (New York: Springer), 336-365.

Lecrux, C., and Hamel, E. (2011). The neurovascular unit in brain function and disease. Acta Physiol. 203, 47-59. doi: 10.1111/j.1748-1716.2011.02256.x

Mahlknecht, P., Hotter, A., Hussl, A., Esterhammer, R., Schocke, M., and Seppi, K. (2010). Significance of MRI in diagnosis and differential diagnosis of Parkinson's disease. Neurodegener. Dis. 7, 300-318. doi: 10.1159/000314495

Mandeville, J. B., Jenkins, B. G., Kosofsky, B. E., Moskowitz, M. A., Rosen, B. R., and Marotta, J. J. (2001). Regional sensitivity and coupling of BOLD and CBV changes during stimulation of rat brain. Magn. Reson. Med. 45, 443-447. doi: 10. 1002/1522-2594(200103)45:3<443::aid-mrm1058>3.0.co;2-3

Markus, H. S., Costa, D. C., and Lees, A. J. (1994). HMPAO SPECT in Parkinson's disease before and after levodopa: correlation with dopaminergic responsiveness. J. Neurol. Neurosurg. Psychiatry 57, 180-185. doi: 10.1136/jnnp.57. 2.180

Marota, J. J. A., Mandeville, J. B., Weisskoff, R. M., Moskowitz, M. A., Rosen, B. R., and Kosofsky, B. E. (2000). Cocaine activation discriminates dopaminergic projections by temporal response: an fMRI study in rat. Neuroimage 11, 13-23. doi: 10.1006/nimg.1999.0520
Money, K. M., and Stanwood, G. D. (2013). Developmental origins of brain disorders: roles for dopamine. Front. Cell. Neurosci. 7:260. doi: 10.3389/fncel. 2013.00260

Moore, C. I., and Cao, R. (2008). The hemo-neural hypothesis: on the role of blood flow in information processing. J. Neurophysiol. 99, 2035-2047. doi: 10.1152/jn. 01366.2006

Morita, K., Morishima, M., Sakai, K., and Kawaguchi, Y. (2013). Dopaminergic control of motivation and reinforcement learning: a closed circuit account for reward-oriented behavior. J. Neurosci. 33, 8866-8890. doi: 10. 1523/JNEUROSCI.4614-12.2013

Nakashima, A., Hayashi, N., Kaneko, Y. S., Mori, K., Sabban, E. L., Nagatsu, T., et al. (2009). Role of N-terminus of tyrosine hydroxylase in the biosynthesis of caecholamines. J. Neural Transm. 116, 1355-1362. doi: 10.1007/s00702-0090227-8

Nguyen, T. V., Brownell, A. L., Iris Chen, Y. C., Livni, E., Coyle, J. T., Rosen, B. R., et al. (2000). Detection of the effects of dopamine receptor supersensitivity using pharmacological MRI and correlation with PET. Synapse 36, 57-65. doi: 10. 1002/(sici) 1098-2396(200004)36:1<57::aid-syn6>3.0.co;2-k

Obeso, J. A., Rodriguez-Oroz, M. C., Benitez-Temino, B., Blesa, F. J., Guridi, J., Marin, C., et al. (2008). Functional organization of the basal ganglia: therapeutic implications for Parkinson's disease. Mov. Disord. 23(Suppl. 3), S548-S559. doi: $10.1002 / \mathrm{mds} .22062$

Paxinos, G., and Watson, C. (1998). The Rat Brain in Stereotaxic Coordinates. Orlando: Academic Press.

Peterson, E. J., and Seger, C. A. (2013). Many hats: intratrial and reward leveldependent BOLD activity in the striatum and promoter cortex. J. Neurophysiol. 110, 1689-1702. doi: 10.1152/jn.00164.2012

Pocotte, S. L., Holz, R. W., and Ueda, T. (1986). Cholonergic receptor-mediated phosphorylation and activation of tyrosine hydroxylase in cultured bovine adrenal chromaffin cells. J. Neurochem. 46, 610-622. doi: 10.1111/j.1471-4159. 1986.tb13011.x

Reinhard, J. F. Jr., Liebmann, J. E., Schlosberg, A. J., and Moskowitz, M. A. (1979). Serotonin neurons project to small blood vessels in the sbrain. Science 206, 8587. doi: $10.1126 /$ science. 482930

Rodríguez, M., Barroso-Chinea, P., Abdala, P., Obeso, J., and Gonzalez-Hernandez, T. (2001). Dopamine cell degeneration induced by intraventricular administration of 6-hydroxydopamine in the rat: similarities with cell loss in Parkinson's disease. Exp. Neurol. 169, 163-181. doi: 10.1006/exnr.2000.7624

Rodríguez-Díaz, M., Abdala, P., Barroso-Chinea, P., Obeso, J., and GonzalezHernandez, T. (2001). Motor behavioural changes after intracerebroventricular injection of 6-hydroxydopamine in the rat: an animal model of Parkinson's disease. Behav. Brain. Res. 122, 79-92. doi: 10.1016/s0166-4328(01)00168-1

Rodriguez-Oroz, M. C., Jahanshahi, M., Krack, P., Litvan, I., Macias, R., Bezard, E., et al. (2009). Initial clinical manifestations of Parkinson's disease: features and pathophysiological mechanisms. Lancet Neurol. 8, 1128-1139. doi: 10. 1016/S1474-4422(09)70293-5

Rodriguez-Perez, A. I., Dominguez-Meijide, A., Lanciego, J. L., Guerra, M. J., and Labandeira-Garcia, J. L. (2013). Dopaminergic degeneration is enhanced by chronic brain hypoperfusion and inhibited by angiotensin receptor blockage. Age 35, 1675-1690. doi: 10.1007/s11357-012-9470-2

Roitman, M. F., Stuber, G. D., Phillips, P. E. M., Wightman, R. M., and Carelli, R. M. (2004). Dopamine operates as a subsecond modulator of food seeking. J. Neurosci. 24, 1265-1271. doi: 10.1523/jneurosci.3823-03.2004

Salin, P., Castle, M., Kachidian, P., Barroso-Chinea, P., López, I. P., Kerkerian-Le Goff, L., et al. (2008). High-resolution neuroanatomical tract-tracing for the analysis of striatal microcircuits. Brain Res. 1221, 49-58. doi: 10.1016/j.brainres. 2008.05.011

Salvatore, M. F., Waymire, J. C., and Haycock, J. W. (2001). Depolarizationstimulated catecholamine biosynthesis: involvement of protein kinases and tyrosine hydroxylase phosphorylation sites in situ. J. Neurochem. 79, 349-360. doi: 10.1046/j.1471-4159.2001.00593.x

Sander, C. Y., Hooker, J. M., Catana, C., Normandin, M. D., Alpert, N. M., Knudsen, G. M., et al. (2013). Neurovascular coupling to D2/D3 dopamine receptor occupancy using simultaneous PET/fuctional MRI. Proc. Natl. Acad. Sci. U S A 110, 11169-11174. doi: 10.1073/pnas. 1220512110

Schultz, W. (2007). Multiple dopamine functions at different time courses. Annu. Rev. Neurosci. 30, 259-288. doi: 10.1146/annurev.neuro.28.061604.135722

Sibarita, J.-B. (2005). Deconvolution microscopy. Adv. Biochem. Eng. Biotechnol. 95, 201-243. doi: 10.1007/b102215 
Tan, C. O. (2009). Anticipatory changes in regional cerebral hemodynamics: a new role for dopamine?. J. Neurophysiol. 101, 2738-2740. doi: 10.1152/jn.00141.2009

Tayebati, S. K., Lokhandwala, M. F., and Amenta, F. (2011). Dopamine and vasculat dynamics control: present status and future perspectives. Curr. Neurovasc. Res. 8, 246-257. doi: 10.2174/156720211796558032

Toda, N. (1976). Influence of dopamine and noradrenaline on isolated cerebral arteries of the dog. Br. J. Pharmacol. 58, 121-126. doi: 10.1111/j.1476-5381.1976. tb07700.x

Van Laere, K., Santens, P., Bosman, T., De Reuck, J., Mortelmans, L., and Dierckx, R. (2004). Statistical parametric mapping of $99 \mathrm{mTc}$-ECD SPECT in idiopathic Parkinson's disease and multiple system atrophy with predominant parkinsonian features: correlation with clinical parameters. J. Nucl. Med. 45, 933-942. Available online at: http://jnm.snmjournals.org/content/45/6/933

Vaucher, E., and Hamel, E. (1995). Cholinergic basal forebrain neurons project to cortical microvessels in the rat: electron microscopic study with anterograde transported Phaseolus vulgaris leucoagglutinin and choline acetyltransferase immunocytochemistry. J. Neurosci. 15, 7427-7441. Available online at: http://www.jneurosci.org/content/15/11/7427.long

Villar-Cheda, B., Sousa-Ribeiro, D., Rodriguez-Pallares, J., Rodriguez-Perez, A. I., Guerra, M. J., and Labandeira-Garcia, J. L. (2009). Aging and sedentarism decrease vascularization and VEGF levels in the rat substantia nigra. Implications for Parkinson's disease. J. Cereb. Blood Flow Metab. 29, 230-234. doi: 10. 1038/jcbfm.2008.127

Willie, C. K., Tzeng, Y.-C., Fisher, J. A., and Ainslie, P. N. (2014). Integrative regulation of human brain blood flow. J. Physiol. 593, 841-859. doi: 10.1113/jphysiol. 2013.268953

Wolfson, L. I., Leenders, K. L., Brown, L. L., and Jones, T. (1985). Alterations of regional cerebral blood flow and oxygen metabolism in Parkinson's disease. Neurology 35, 1399-1405. doi: 10.1212/wnl.35.10.1399

Xu, Z.-Q., Lew, J. Y., Harada, K., Aman, K., Goldstein, M., Deutch, A., et al. (1998). Immunohistochemical studies on phosphorylation of tyrosine hydroxylase in central catecholamine neurons using site- and phosphorylation state-specific antibodies. Neuroscience $82, \quad 727-738$. doi: 10.1016/S0306-4522(97) 00189-9

Yung, K. K., Bolam, J. P., Smith, A. D., Hersch, S. M., Ciliax, B. J., and Levey, A. I. (1995). Immunocytochemical localization of D1 and D2 dopamine receptors in the basal ganglia of the rat: light and electron microscopy. Neuroscience 65, 709-730. doi: 10.1016/0306-4522(94)00536-e

Zanassi, P., Paolillo, M., Montecucco, A., Avvedimento, E. V., and Schilelli, S. (1999). Pharmacological and molecular evidence for dopamine $\mathrm{D}(1)$ receptor expression by striatal astrocytes in culture. J. Neurosci. Res. 58, 544-552. doi: 10. 1002/(sici)1097-4547(19991115)58:4<544::aid-jnr7>3.0.co;2-9

Zigmond, M. J., and Stricker, E. M. (1973). Recovery of feeding and drinking by rats after intraventricular 6-hydroxydopamine or lateral hypothalamic lesions. Science 182, 717-720. doi: 10.1126/science.182.4113.717

Conflict of Interest Statement: The authors declare that the research was conducted in the absence of any commercial or financial relationships that could be construed as a potential conflict of interest.

Received: 13 June 2014; accepted: 02 August 2014; published online: 26 August 2014. Citation: Afonso-Oramas D, Cruz-Muros I, Castro-Hernández J, Salas-Hernández J, Barroso-Chinea P, García-Hernández S, Lanciego JL and González-Hernández $T$ (2014) Striatal vessels receive phosphorylated tyrosine hydroxylase-rich innervation from midbrain dopaminergic neurons. Front. Neuroanat. 8:84. doi: $10.3389 /$ fnana.2014.00084

This article was submitted to the journal Frontiers in Neuroanatomy.

Copyright (c) 2014 Afonso-Oramas, Cruz-Muros, Castro-Hernández, SalasHernández, Barroso-Chinea, García-Hernández, Lanciego and González-Hernández. This is an open-access article distributed under the terms of the Creative Commons Attribution License (CC BY). The use, distribution or reproduction in other forums is permitted, provided the original author(s) or licensor are credited and that the original publication in this journal is cited, in accordance with accepted academic practice. No use, distribution or reproduction is permitted which does not comply with these terms. 